\title{
1 A wind tunnel for the calibration of Mars wind sensors
}

2 C. F. Wilson * (1), A. L. Camilletti (1), S. B. Calcutt (1), P. M. Ligrani (2)

3 (1) Dept of Physics, Oxford University

4 (2) Dept of Engineering Science, Oxford University

6 Abstract:

7 A major limitation in the development of wind sensors for use on Mars is the lack of suitable

8 testing and calibration facilities. A low density wind tunnel has been developed at Oxford

9 University for calibration of wind sensors for Mars landers, capable of providing stable or

10 dynamically varying winds, of air or carbon dioxide, at Martian pressures (5 - 10 mbar) and

11 speeds $(0.5-30 \mathrm{~m} / \mathrm{s})$, and temperatures of $200-300 \mathrm{~K}$. The flow field in the test section was

12 calculated using analytical and computational modelling techniques, and validated

13 experimentally using a pitot probe. This facility's stability and accuracy offer significant

14 advantages with respect to previous calibration facilities.

15 Keywords: Mars; Martian meteorological sensors; Wind tunnel; laminar flow; anemometer;

16 calibration

* Corresponding author 


\section{$17 \quad 1$ Introduction}

\section{$18 \quad 1.1$ Science Rationale}

19 Measuring the near-surface winds on Mars is critical for a number of reasons. Near-

20 surface winds represent a boundary condition for the rest of the atmospheric

21 circulation; it is at the surface that most of the heat input to the atmosphere occurs; it

22 is here that sources and sinks of volatiles like water and methane occur; and,

23 particularly, near-surface winds determine the surface-atmosphere exchanges of dust,

24 governing the onset and subsidence of dust storms. Near-surface winds are also

25 critical from an engineering point of view for future exploration programmes, both

26 for safe landing and for continued operation of landers and/or aerobots.

27 Measurement of wind on Mars is difficult, due largely to the low density of the

28 atmosphere. Many different techniques of wind measurement on Mars have been

29 proposed, including dynamic pressure devices like 'windsocks' (Sullivan et al., 2000)

30 and telltales (Gunnlaugsson et al., 2006), to ion drift devices (Evlanov et al., 2001),

31 laser-based devices (Merrison et al., 2006), and ultrasonic devices (Dissly et al.,

32 2005). All wind data from Mars to date, though, has come from thermal wind sensors

33 on the Viking landers (Chamberlain et al., 1976) and the Mars Pathfinder lander

34 (Seiff et al., 1997); thermal wind sensors are also baselined for the future Mars

35 Science Laboratory (MSL) and ExoMars missions due to their simplicity of

36 construction and robustness.

37 However, thermal wind sensors are sensitive to any change in thermal conditions, in

38 particular to changes in air temperature. On Mars, during the daytime, the ground 
39 rapidly warms up in the sun, setting up a strong temperature gradient in the lowest

40 few metres of the atmosphere. This temperature inhomogeneity is then advected by

41 atmospheric turbulence, leading to rapid and frequent temperature swings at a given

42 location. This can be seen in the wind and temperature data from Viking Lander 2

43 shown in Figure 1. Data from Viking Lander 2 and Mars Pathfinder missions show

44 that temperature swings of $10^{\circ} \mathrm{C}$ can occur over periods as short as 10 seconds.

45 Because thermal wind sensors are particularly sensitive to swings in air temperature

46 (as well as other thermal loads such as sunlight or inhomogeneity in thermal infrared

47 loads), it is important that these can be replicated in test facilities. For example,

48 calibrated wind data have still not been published for the Mars Pathfinder thermal

49 wind sensor due to uncertainties in thermal conditions at the wind sensor. The

50 development of this and other thermal wind sensors has been hampered by a lack of

51 calibration facilities.

52 From the previous discussion, it is evident that there are no standard wind sensors

53 available for use to calibrate test facilities. Laser-Doppler anemometry is currently

54 used as a standard in some wind tunnels; however, this requires airborne particles in

55 the flow, which is not always consistent with flight hardware cleanliness

56 requirements. In this paper, we discuss different approaches which can be used to

57 simulate Martian winds, highlighting the advantages and disadvantages of the

58 different methods. We then describe in greater detail a wind tunnel developed

59 specifically for the calibration of wind sensors for Mars. 


\subsection{Turbulent or laminar flow?}

61 The first task in any wind tunnel design is to consider the Reynolds number of the

62 flow - this characterizes the level of turbulence to be expected in the flow. The

63 Reynolds number is given by $R e=u D \rho / \mu$, where $u$ is flow velocity, $D$ is a length

64 (typically, the diameter of the wind tunnel test section), and $\rho$ and $\mu$ represent

65 respectively the density and dynamic viscosity of the fluid. In the rarefied Martian

66 atmosphere, any wind tunnel with a diameter of order $1 \mathrm{~m}$ is likely to exhibit

67 turbulent flow, because it will have a Reynolds number in excess of $10^{4}$ for speeds

68 above $10 \mathrm{~m} / \mathrm{s}$. This suggests the use of a wind tunnel $<0.5 \mathrm{~m}$ in diameter, in order to

69 keep the Reynolds numbers below $10^{4}$ and thus make it possible to achieve laminar

70 flow.

71 In addition, the boundary layer at the wind tunnel walls is of greater thickness in a

72 low density atmosphere than in the equivalent flow at higher density (the theoretical

73 basis for this is discussed below in Section 2.1). Therefore, if the aim is to test in the

74 central region of the flow, uninfluenced by boundary layers from either wall of the

75 wind tunnel, it is useful to have a small length-to-width ratio. In summary, a large

76 wind tunnel with a large length-to-width ratio will always exhibit large-eddy

77 turbulence, especially at higher speeds.

78 Two leading Mars wind tunnels exhibit turbulent flow - the Danish Mars Wind

79 Tunnel at Aarhus University, which is $0.4 \mathrm{~m}$ diameter x $1.5 \mathrm{~m}$ long (Merrison et al.

80 2002), and the Mars Wind Tunnel (MARSWIT) at NASA Ames Research Centre

81 (e.g. White et al., 1997; Greeley et al., 2000), which is $1.3 \mathrm{~m} \mathrm{x} 1.3 \mathrm{~m}$ in cross section

82 and $13 \mathrm{~m}$ long. Turbulence in the wind tunnel is useful for studying dust transport 
83 due to boundary layer turbulence, which is a particular focus for these two facilities,

84 but not helpful for calibration of wind sensors. For this task it is preferable to have

85 laminar flow in the wind tunnel, which is achieved by using a laminar flow nozzle, as

86 will be explained below. As discussed above, the length of the wind tunnel must be

87 kept relatively short in order to keep the boundary layer development to a minimum.

88 This approach was used for calibration of the Viking Wind sensor (Henry \& Greene,

89 1974), and is adopted again for the Oxford Mars wind tunnel.

$91 \quad 1.3$ Rotating arm, recirculating, or open-circuit wind tunnel?

92 Wind tunnels can be subdivided broadly into three categories, as shown in Figure 2;

93 rotating arm, re-circulating, and open-circuit.

94 A rotating arm is perhaps the oldest form of wind testing facility, and is still used

95 today thanks to its simplicity and low cost. A Mars wind sensor test facility at

96 NASA's Jet Propulsion Laboratory (JPL) uses a rotating arm with a length of $1 \mathrm{~m}$,

97 rotating in a $3 \mathrm{~m}$ diameter chamber (D. Crisp, personal communication). Speeds

98 obtainable in this facility are limited, for aerodynamic and mechanical reasons, to a

99 maximum of $10 \mathrm{~m} / \mathrm{s}$, at which speed the arm is rotating at $\sim 1.5$ revolutions per

100 second. Also, the centripetal acceleration inherent in rotating arm systems makes

101 them unsuitable for use in calibrating mechanical wind sensors.

102 Another approach is to use a recirculating wind tunnel. In this case the entire system

103 is contained within an environmental chamber, in which the required pressure and

104 temperature are maintained; within this, one can construct a standard wind tunnel 
105 using a fan and ducting to circulate the gas past the test piece. A Mars wind tunnel at

106 the University of Aarhus in Denmark is of this type, and has a test section of $0.4 \mathrm{~m}$

107 diameter x $1.5 \mathrm{~m}$ long. This facility typically exhibits turbulent flow, which is well-

108 suited to its primary focus on studies of dust and sand transport; this turbulence

109 causes fluctuations in the wind speed quoted as ranging from $4 \%$ to around $16 \%$

110 (Merrison et al., 2006).

111 A disadvantage of all the re-circulating wind tunnels is that it is difficult to introduce

112 sudden changes in either wind speed or temperature. One solution to this problem is

113 to feed the test section with an external gas supply, with the flow speed governed by

114 a high-pressure drop. Once it has passed through the test section, the gas is then

115 evacuated using vacuum pumps. This again follows the approach used for Viking

116 wind sensor calibration by Henry \& Greene (1974), as mentioned above.

117 A hybrid approach is used by the MARS WInd Tunnel (MARSWIT), located at the

118 NASA Ames Research Centre, which has a test section is $1.3 \mathrm{~m} \mathrm{~W}$ x $1.3 \mathrm{~m} \mathrm{H}$ x $13 \mathrm{~m}$

119 in length (see e.g. Greeley et al., 2000). MARSWIT is an open-circuit wind tunnel

120 located within a large $\left(\sim 4000 \mathrm{~m}^{3}\right)$ vacuum chamber. At Martian pressures, wind is

121 generated by injecting high pressure air or $\mathrm{CO}_{2}$ at the exit of wind tunnel such that

122 suction is generated. This wind tunnel thus has the advantage of being capable of

123 relatively rapid changes in velocity (thanks to the gas injection system), and yet has

124 the advantages of stability in composition and temperature because it is located

125 entirely within a large test chamber. Like the Danish Mars wind tunnel, MARSWIT

126 has a high length-to-width ratio, in order to let the turbulent boundary layer develop.

127 This is ideal for surface boundary layer and Aeolian studies, but make it ill-suited to

128 calibration of wind sensors (see for example Seiff et al., 1997). 


\section{Oxford Mars wind tunnel: design}

131 An open-circuit wind tunnel has been created, as is shown in Figures 3 and 4, and is

132 called the Mars Environment Wind Tunnel. The present wind tunnel is an adaptation

133 of a previously existing facility, the Low Density Wind Tunnel (LDWT), at the

134 Southwell Laboratory of Oxford University's Department of Engineering Science.

135 The LDWT already operated as an open-circuit wind tunnel of the type described

136 above, so it was possible to use without modification the large vacuum chamber itself

137 and its powerful vacuum pumping system. The flow conditions required for this work

138 (cool subsonic gas at a few millibars) are very different from those of the original

139 LDWT (heated hypersonic gas at a few microbars), so several new sub-systems had

140 to be implemented. The designs of the different subsystems of the wind tunnel are 141 described below.

\section{$142 \quad 2.1$ Nozzle design}

143 The optimum diameter of the wind tunnel's test section was obtained by dividing the

144 maximum possible pumping speed, conservatively estimated at $\sim 0.84 \mathrm{~m}^{3} \mathrm{~s}^{-1}$, by the

145 maximum flow speed needed $(30 \mathrm{~m} / \mathrm{s})$. This gives a test section area of $\sim 0.03 \mathrm{~m}^{2}$,

146 corresponding to a test section of diameter $D=0.18 \mathrm{~m}$. For carbon dioxide at

147 pressures of $6-10$ mbar and temperatures of $200-300 \mathrm{~K}$, the gas density ranges

148 from 3 to $9 \mathrm{~g} \mathrm{~m}^{-3}$. Thus we can calculate that the Reynolds numbers of the flow will

149 range from 80 to 10000 . At lower Reynolds numbers the flow will clearly be laminar; 
150 however, a well-designed nozzle is required in the range $10^{3}<R e_{D}<10^{4}$ to ensure

151 uniform and laminar flow in the test section.

152 When gas enters the vacuum chamber, it is travelling at near-sonic velocity in a 153 small-bore pipe, and is very turbulent. It first enters a settling chamber, where the gas 154 decelerates, and its pressure equilibrates at near-Martian pressure. A deflector plate is 155 put at the entrance to this chamber in order to facilitate deceleration and mixing of 156 the gas. The gas then passes through a series of screens. The first screen is a porous 157 sintered plastic plate (pore size $100 \mu \mathrm{m}$; thickness $3.2 \mathrm{~mm}$ ); this ensures an even 158 distribution of airflow across the cross-section of the wind tunnel. Parallel flow is 159 ensured by passing the flow through a honeycomb flow straightener ( $5 \mathrm{~mm}$ cell width $160 \times 50 \mathrm{~mm}$ length), and then any residual vortices are broken into smaller cells by a 161 final wire mesh with $1 \mathrm{~mm}$ spacing. At this point the diameter of the flow is still 400 $162 \mathrm{~mm}$, greater than the $180 \mathrm{~mm}$ test section diameter. This larger diameter ensures that 163 the flow is still slow when the flow passes through the flow straightener and mesh, 164 which ensures that turbulent eddies can be quickly dissipated, resulting in laminar 165 flow even at the highest test speeds. Finally, a contracting nozzle is used to reduce 166 the flow diameter from $400 \mathrm{~mm}$ to $180 \mathrm{~mm}$, accelerating the flow smoothly by a 167 factor of $\sim 5$. The test section, i.e. the location in the wind tunnel where the wind 168 sensor is placed, is simply at the outlet of the nozzle. A nozzle with a circular section 169 was used in preference to a rectangular one because it does not have corners, which 170 can introduce inhomogeneity into the flow.

171 The shape of the nozzle itself was based on established nozzle design principles; the 172 rate of change of the nozzle's cross section is rapid at first, then less so as the fluid is 173 accelerated (e.g. Barlow, 1999). The length of the nozzle is kept fairly short, so that 
174 the boundary layer thickness is still small when the flow reaches the test section. The 175 nozzle shape was chosen following finite-element modelling (FEM) calculations 176 using a commercial Computational Fluid Dynamics (CFD) package (Fluent). The 177 mesh used for the FEM calculation was a two-dimensional (axisymmetric) model, 178 with a higher density of nodes in the boundary layer. The mesh used had $40 \times 40$ 179 nodes; it was found that higher node densities did not significantly change the results 180 (mesh densities of up to $120 \times 60$ elements were tried, which changed local flow 181 velocities by less than $0.2 \%$ ). A uniform inlet velocity was specified as a boundary 182 condition. The output parameter of most interest is the radial profile of axial velocity 183 at the test section, i.e. at the exit of the nozzle.

184 Flow profiles were calculated for various flow speeds ranging from $0.1 \mathrm{~m} / \mathrm{s}$ to 20 $185 \mathrm{~m} / \mathrm{s}$. These flow profiles are presented in Figure 5 as a proportion of $u_{\text {ave }}$, where $u_{\text {ave }}$

186 is the averaged flow speed in the section $\left(u_{\text {ave }}=\int_{0}^{R} u(r) 2 \pi r d r\right)$. These calculations 187 assumed that the test gas was carbon dioxide at $p=6$ mbar and $T=250 \mathrm{~K}$. At all 188 speeds above $1 \mathrm{~m} / \mathrm{s}$, the variation in velocity across the central $45 \mathrm{~mm}$ of the test 189 section is under $1 \%$; at all speeds above $3.5 \mathrm{~m} / \mathrm{s}$, the variation in velocity across the 190 central $45 \mathrm{~mm}$ of the test section is under $0.5 \%$.

191 In order to validate and to understand better the FEM results, the velocity profile was 192 also studied analytically by calculating boundary layer development. The boundary 193 layer at the wall of the wind tunnel is expected to be laminar in all flow conditions. If 194 the Reynolds number is calculated using the length of the wall $(x \sim 0.45 \mathrm{~m})$, it stays 195 well below $3 \times 10^{5}$ - commonly accepted as a guideline value for the onset of 196 turbulence in this case - for all test conditions. The displacement thickness of the 
197 boundary layer was calculated using the Thwaites method for boundary layer

198 development in accelerating flow (see e.g. Young, 1989). This states that the

199 boundary layer momentum thickness $\theta$ is given by

200

$$
\theta^{2}=0.45 v u^{-6} \int_{0}^{x}[u(x)]^{5} d x
$$

201 where $u(x)$ is the free stream flow velocity as a function of $x$, the distance along the 202 wall, and $v$ is the dynamic viscosity of the fluid $(v=\mu / \rho)$. The value of the integral on 203 the right is proportional to $u^{5}$. Therefore the boundary layer thickness scales as $204 \theta^{2} \sim v / u$, i.e. $\theta \sim R e^{-1 / 2}$. Therefore the profile calculated for any given flow conditions 205 should be the same as that for any other flow conditions with the same Reynolds 206 number.

207 Given the displacement thickness and the free stream flow velocity, the expected 208 velocity profile in the boundary layer can be obtained using a numerical solution to 209 the Blasius equation, as shown for example in Fay and Sonwalkar (1991). It is

210 assumed that the flow is uniform in the centre of the nozzle, as long as the boundary

211 layer thickness is significantly less than the radius of the nozzle, an assumption 212 which breaks down at low speeds. Calculated velocity profiles are in good agreement 213 with FEM results at $u>3 \mathrm{~m} / \mathrm{s}$, as will be shown below.

214 At very low velocities $\left(R e_{D} \leq 300\right.$, corresponding roughly to $u \leq 2 \mathrm{~m} / \mathrm{s}$ ), the 215 displacement thickness becomes significant in comparison to the radius of the wind 216 tunnel. In this case the flow profile approaches Poiseuille flow, i.e. fully developed 217 laminar pipe flow, which has a quadratic dependence on radius. 
218 This primary nozzle, with its diameter of $0.18 \mathrm{~m}$, is designed for flow speeds ranging

219 from $\sim 0.5$ to $30 \mathrm{~m} / \mathrm{s}$. A second nozzle is also sometimes used, with a diameter of

$2200.12 \mathrm{~m}$, which allows flow speeds of up to $65 \mathrm{~m} / \mathrm{s}$ (due to its smaller cross-sectional

221 area). A third possibility is to remove the nozzle entirely, which permits operation at

$2220.1-6 \mathrm{~m} / \mathrm{s}$. At such low flow speeds, flow will be laminar with or without the

223 aerodynamic nozzle. This also allows a large test section (400 $\mathrm{mm}$ in diameter), and

224 removes the problem of the growing boundary layer which causes uncertainty in the

225 exact flow speed at the test piece. A similar approach (a porous plug without a

226 contoured nozzle) was used for low speed flow in the temporary Viking wind sensor

227 test facility at NASA’s Langley Research Centre (Henry \& Greene, 1974).

228 In summary, the wind tunnel may be used with either a nozzle of $180 \mathrm{~mm}$ diameter,

229 or with a smaller nozzle of $120 \mathrm{~mm}$ which allows higher flow speeds. The nozzles

230 have been designed to produce laminar flow, in order to minimise spatial and 231 temporal velocity non-uniformities in the test section, at speeds of up to $30 \mathrm{~m} / \mathrm{s}$ (or up

232 to $60 \mathrm{~m} / \mathrm{s}$ for the $120 \mathrm{~mm}$ nozzle). The spatial non-uniformity in the central $2 \mathrm{~cm}$ of

233 the flow field has been predicted, using finite-element modelling (FEM), to be less

234 than $1 \%$ for all flow speeds above $1 \mathrm{~m} / \mathrm{s}$ (or $2 \mathrm{~m} / \mathrm{s}$ for the $120 \mathrm{~mm}$ nozzle).

\section{$235 \quad 2.2$ Gas flow and pressure control}

236 The wind tunnel can be run using either air or carbon dioxide. A large $\left(\sim 10 \mathrm{~m}^{3}\right)$

237 balloon, filled with $\mathrm{CO}_{2}$ at room pressure, is used as the source reservoir. This system

238 is used rather than using $\mathrm{CO}_{2}$ directly from gas cylinders for two reasons. The main

239 reason is that, at the high flow rates used in the wind tunnel, $\mathrm{CO}_{2}$ would freeze the

240 regulators in the flow path. This possibility is commonly avoided by heating the $\mathrm{CO}_{2}$ 
241 immediately before it passes through the regulators, but this leaves one with warm

242 gas, the temperature of which would be dependent on the flow rate being used.

243 A single valve, immediately upstream of the pressure chamber, is used to set the

244 mass flow rate. All gas upstream of this flow control valve is at, or near, atmospheric

245 pressure; downstream of this valve, the gas is at or near Martian pressure ( $\sim 6$ mbar),

246 or in fact slightly higher due to pressure gradients along the short pipe connecting

247 this valve to the test chamber. In normal operation, then, this valve acts as a critical

248 orifice, such that the flow rate and upstream pressure are independent of the

249 downstream pressure, because the upstream-to-downstream pressure ratio at this

250 valve is less than 0.53 (Parkinson and Day, 1979). Using an ordinary rubber weir-

251 type vacuum valve for this purpose, it was found that the mass flow rate could be set

252 to a resolution of $\sim 0.1 \%$ of the maximum flow rate (i.e. roughly $0.03 \mathrm{~m} / \mathrm{s}$ ), which is

253 more than adequate.

254 Once the mass flow through the wind tunnel is set, the chamber pressure is set by 255 using a valve immediately upstream of the vacuum pumps to provide an extra load on 256 the vacuum pumps by admitting air from the atmosphere (as shown at the right of 257 figure 3). Opening this valve increases the chamber pressure, and closing it decreases 258 the chamber pressure. This pressure control valve is controlled with a stepper motor 259 using a commercial proportional-integral-derivative (PID) controller, with adaptive 260 tuning. This works very well in practice, keeping the pressure constant to an accuracy 261 of \pm 0.01 mbar without operator intervention. The PID loop has proved very effective 262 at dealing with sudden changes in load conditions, such as when the wind speed is 263 changed, typically regaining the equilibrium pressure within 10-20 seconds. The 264 pressure in the test chamber is measured using a capacitive transducer (MKS 
265 Baratron, 0-10 torr range), whose calibration certificate shows it to have an accuracy 266 of 0.005 mbar.

\section{$267 \quad 2.3$ Mass flow rate monitoring.}

268 The primary measurement of the wind speed in the tunnel is obtained by measuring

269 the mass flow rate of gas entering the tunnel. This is constantly monitored by 270 measuring the pressure drop across an orifice plate, which is (to a first

271 approximation) proportional to the square of the flow rate. The flow rates in the wind

272 tunnel vary from $\sim 0.1$ to $10 \mathrm{~g} / \mathrm{s}$, so the differential pressure sensors must be capable

273 of accurately reading pressures varying over 4 orders of magnitude. Therefore two

274 orifice plates are used to cover the full range, respectively $6 \mathrm{~mm}$ and $14 \mathrm{~mm}$ in

275 diameter. The larger orifice is used for higher flow rates (mass flow $>3 \mathrm{~g} / \mathrm{s}$ ), and is

276 monitored using a single 0-100 mbar differential pressure sensor (SensorTechnics

277 HCXM series). The smaller orifice plate is monitored using two differential pressure

278 sensors, with ranges of $0-10$ mbar and 0-100 mbar respectively. This leads to a

279 differential pressure measurement accuracy of $<1 \%$ of the reading, across the whole 280 range of flow rates.

281 The flow rate is determined using British Standard (BS) 1042, Sections 1:1, 1:2, 1:4.

282 According to the BS, the absolute accuracy of this method of flow rate determination

283 is $1 \%$ to $2 \%$. The absolute accuracy of the mass flow determination in the wind 284 tunnel is thus estimated at $2 \%$ to $3 \%$. The resolution of the system is better than this, 285 though - the repeatability of the differential pressure sensors is $0.25 \%$ of their full286 scale range (limited at present by the resolution of the analogue-to-digital converter 
287 used to record their output). Thus the precision to which flow conditions can be

288 repeated is better than $1 \%$ of flow speed, for all flow speeds above $\sim 1 \mathrm{~m} / \mathrm{s}$.

289 Note that the orifice plates allow determination of the total mass flow rate into the

290 wind tunnel; if one divides this by the fluid density and by the cross-sectional area of

291 the wind tunnel one can calculate the average velocity in the wind tunnel, $u_{\text {ave }}$. The

292 local flow velocity at any point in the test section is then deduced from the modelling

293 results presented in Figure 5. However, these results should be verified using local

294 flow speed measurements in the test section

\section{$295 \quad 2.4$ Local flow speed measurements}

296 In order to characterise the spatial and temporal variations of velocity in the test 297 section of the wind tunnel, one needs a method of measuring local flow velocity. In 298 several other Mars wind tunnels, local flow velocity is measured through laser299 doppler anemometry (LDA), using dust particles suspended flow. Dust cannot be 300 used in the Oxford facility because it would damage the vacuum pumps. In principle, 301 LDA could be used with oil droplets or smoke particles, but this is not compatible 302 with cleanliness requirements in the facility.

303 To characterise the flow profiles in the wind tunnel, we used a pitot tube mounted on 304 a three-dimensional translation stage to enable it to be moved throughout the test 305 section. This incorporates both static tappings (which face perpendicular to the flow 306 direction, thus measuring the absolute ambient pressure), and a pitot probe (facing 307 into the flow). The difference between the static and the pitot pressure is the dynamic 308 pressure, equal to $p_{d y n}=1 / 2 \rho u^{2}$. The density $\rho$ is well known because the chamber 309 pressure and temperature are being measured, which means that the speed $u$ can be 
310 calculated on the basis of the dynamic pressure measurement. The density of the gas

311 is accurately known (to better than $0.1 \%$ ), so the pitot probe offers an absolute

312 measurement of local flow velocity with which to calibrate the wind tunnel.

313 The dynamic pressure is extremely low in the Martian atmosphere, due to a

314 combination of low density and low wind speeds $\left(p_{d y n}<10 \mathrm{~Pa}\right.$ for all wind speeds

315 below $30 \mathrm{~m} / \mathrm{s}$ ). The most sensitive differential pressure sensor commercially

316 available (Furness Controls FCO-332) has a full-scale sensitivity of $20 \mathrm{~Pa}$ and an

317 accuracy of $0.03 \mathrm{~Pa}$. Therefore, an accuracy of $10 \%$ or above in wind speed

318 measurement can only be obtained for wind speeds of $6 \mathrm{~m} / \mathrm{s}$ and above, for $\mathrm{CO}_{2}$ at 7

319 mbar and $250 \mathrm{~K}$. This inability to use the pitot tube at low wind speeds emphasizes

320 once again that the dynamic pressures associated with light winds on Mars are

321 extremely small and difficult to measure.

322 Another important source of error when using the pitot probe in this wind tunnel is

323 fluctuations in the static pressure of the chamber, i.e. the ambient pressure of the gas.

324 These fluctuations are the result of the dynamic equilibrium between the pumping

325 speed and the gas inlet speed. The pressure control system can hold the chamber

326 pressure steady to within $1 \mathrm{~Pa}$, i.e. $0.01 \mathrm{mbar}$, which is stable enough for most

327 purposes of environmental simulation. However, these fluctuations in static pressure

328 are large compared to the dynamic pressures of $0.3-10 \mathrm{~Pa}$ measured at the pitot

329 tube; this is a problem because the dynamic pressure being measured is the difference

330 between the static pressure and the total pressure in the pitot tube. To give an

331 estimate of the importance of this error: a fluctuation of $0.1 \mathrm{~Pa}$ due to static pressure

332 fluctuations gives rise to an error of $1.3 \mathrm{~m} / \mathrm{s}$ if the flow speed is $5 \mathrm{~m} / \mathrm{s}$, and $0.2 \mathrm{~m} / \mathrm{s}$ if

333 the flow speed is $30 \mathrm{~m} / \mathrm{s}$ (These errors have been calculated assuming $\mathrm{CO}_{2}$ at $p=7$ 
334 mbar and $T=250 \mathrm{~K}$ ). This source of error can be minimised by averaging over

335 several readings (over $\sim 20$ seconds); however, it does mean that the pitot probe

336 cannot be used to characterise the temporal variability of the flow speed. The

337 temporal variability was characterised using a hot-film probe, as will be discussed in

338 the next section.

339 Two flow profiles obtained using the pitot probe are shown in Figure 6. These 340 profiles are for air at $T=299 \mathrm{~K}$ and $p=10 \mathrm{mbar}$, for flow speeds of 6.1 and $32.1 \mathrm{~m} / \mathrm{s}$

341 (nominal flow speed in the centre of the test section, as calculated analytically).

342 Analytical and finite-element modelling (FEM) results are included as well as

343 experimental results; it can be seen that all three are in good agreement. The flow

344 enhancement in the centre of the test section at high speed of about $1 \mathrm{~m} / \mathrm{s}$, seen in

345 Figure 6, is not seen in the FEM results. This was due to use of a porous deflector

346 (see Figure 3), which allowed a jet of air through at the centre of the test section. This

347 has since been corrected by using a non-porous deflector.

348 A two-dimensional velocity profile of the test section is shown in Figure 7, obtained

349 by scanning the pitot probe through the test section on a motorised translation stage.

350 It can be seen that the velocity is uniform to $\sim 3 \%$ over the central region of the test

351 section. It is emphasized though that this constraint of $3 \%$ on the uniformity of the

352 velocity field is due to the sensitivity of the velocity measuring technique to chamber

353 pressure fluctuations as discussed above (due to the number of data points taken in

354 this scan, it was not possible to time-average many readings for each data point).

355 Thus it is suspected that the real velocity non-uniformity across the central area of the

356 test section is less than $3 \%$, quite possibly less than $1 \%$ as predicted by the FEM 357 results. 


\section{$358 \quad 2.5 \quad$ Temporal stability}

359 The present wind tunnel has been designed to produce laminar flow even at high

360 wind speeds. In this section of the paper we consider first the theoretical basis of this

361 statement and then discuss experimental evidence for fluctuations in the flow speed.

362 Let us consider the flow when it passes through the final mesh upstream of the test

363 section. The final mesh has a wire spacing of $1 \mathrm{~mm}$, so this is the maximum eddy

364 size which can be expected in the flow at this point. The flow here is travelling five

365 times more slowly than in the test section, because it has not yet passed through the

366 accelerating nozzle. Therefore the Reynolds number here reaches a maximum of 16

367 (calculated by assuming $\mathrm{CO} 2$ at $10 \mathrm{mbar}$ and $200 \mathrm{~K}$, at a flow speed of $6 \mathrm{~m} / \mathrm{s}$ and

368 using $\mathrm{L}=1 \mathrm{~mm}$ representing the mesh spacing). At these low Reynolds numbers, the

369 energy in these eddies quickly dissipates through viscous processes. In any case, this

370 dimension of $1 \mathrm{~mm}$ is smaller than any of the wind sensors under consideration for

371 future use on Mars so are expected to be undetectable. However, in order to test this

372 hypothesis we have looked for fluctuations in the flow speed experimentally.

373 As described above, the pitot tube could not be used to measure temporal variations

374 of local flow velocity due to its sensitivity to static pressure fluctuations. In order to

375 characterise temporal fluctuations in the local flow speed, then, we need to use a

376 different technique. For this purpose we used a hot-film anemometry probe which

377 was a flight spare wind sensor from the Viking Lander, kindly provided to us by Jim

378 Tillman of the University of Washington. This consisted of a platinum film deposited

379 onto the surface of a quartz cylinder, $0.5 \mathrm{~mm}$ in diameter x $10 \mathrm{~mm}$ long (Davey et al.,

380 VMIS design report, 1973). The Viking wind sensor electronics were not available, 
381 so the sensor was driven using a commercial constant-temperature anemometry

382 (CTA) bridge (Dantec 55M10). We obtained a calibration curve to relate power

383 dissipation to wind speed. Because this calibration curve was obtained with respect to

384 the wind tunnel's measurement of wind speed, we can not use the Viking wind

385 sensor as an absolute calibration standard for the Oxford wind tunnel. However, the

386 calibration curve does permit us to quantify the magnitude of velocity fluctuations in

387 the wind tunnel.

388 Time series of velocity measurements, obtained with the hot film probe positioned in 389 the centre of the test section, are presented in Figure 8 for flow velocities of 6.7 and $39025.4 \mathrm{~m} / \mathrm{s}$. The y error bars represent the sampling resolution limit of the analog-to-

391 digital converter used to record the data. (each reading represents the average of 100

392 readings taken over the interval of $200 \mu \mathrm{s}$; this was routinely performed on all ADC

393 channels to reduce electrical noise). It can be seen that, at frequencies of $<1 \mathrm{~Hz}$, the 394 velocities appear stable to within $0.1 \%$.

395 If there is any turbulence in the flow, though, it would be expected to be at higher 396 frequencies. When its output was measured with an oscilloscope, the thermal wind 397 sensor was found to be sensitive to turbulence at up to $5 \mathrm{kHz}$ (this was found by 398 placing it in a turbulent boundary layer). However, no measurable fluctuations in 399 flow speed were found when the wind sensor was placed in the centre of the wind 400 tunnel's test section. Based on the sensitivities of the equipment used, this places an 401 upper bound of $1 \%$ on velocity fluctuations in the centre of the wind tunnel for 402 velocities over $5 \mathrm{~m} / \mathrm{s}$. 
403 Another measure of temporal stability of the flow in the wind tunnel comes from the

404 orifice plate flow meters. At high flow speeds, the flow rate at the orifice plates is 405 occasionally observed to fluctuate by $\sim 0.3 \%$, perhaps due to turbulence at the flow 406 control valve (this valve emits a 'whistle' which fluctuates slightly in pitch at high 407 flow rates). This fluctuation is only large enough to be detectable at $u>20 \mathrm{~m} / \mathrm{s}$; at 408 this speed the fluctuations are only $\sim \pm 0.06 \mathrm{~m} / \mathrm{s}$, i.e. $\sim \pm 0.3 \%$. This is a very small 409 fluctuation, and represents a significantly smaller fluctuation than would be 410 encountered in turbulent wind tunnels (c.f. $4 \%$ to $16 \%$ quoted for the Aarhus wind 411 tunnel (Merrison et al., 2006).

\section{$412 \quad 2.6 \quad$ Temperature control}

413 Gas entering the test section can be cooled by passing it through a heat exchanger

414 immediately before it enters the wind tunnel. Cooling power is provided by a closed415 cycle cascade cooler (PolyCold PFC1100), which is capable of delivering $1 \mathrm{~kW}$ of 416 cooling power at $-100^{\circ} \mathrm{C}$. The temperature of the heat exchanger is controlled using 417 another PID loop to switch the cooling circuit on and off. The minimum temperature 418 at which the wind tunnel can be run is around $200 \mathrm{~K}$; this is limited by heat losses 419 from the nozzle assembly (these are reduced by covering it with an insulating 420 blanket, not shown in Fig. 3). In future, it may be possible to run at temperatures 421 down to $170-180 \mathrm{~K}$ by pre-cooling the reservoir and nozzle, although it will always 422 be difficult to operate so close to the freezing temperature of carbon dioxide.

423 Although the gas stream itself is cooled, the test chamber itself remains at room 424 temperature. This has two implications for testing. First of all, it means that one 425 cannot simulate cold conditions with zero wind: as soon as the wind is turned off, 
426 anything in the test section warms up, through a combination of radiative and

427 convective heat transfer. Secondly, it means that the thermal IR radiative background

428 on any object in the test section has a brightness temperature of $\sim 293 \mathrm{~K}$ rather than

429 the $150-250 \mathrm{~K}$ which might be more appropriate for Mars. This second effect is

430 especially important to consider for thermal wind sensors. This thermal IR

431 background temperature could be controlled in future by incorporating a thermal

432 shroud with an independent cooling loop, which would allow independent control of

433 the effective thermal IR background temperature; however, this has not yet been

434 implemented.

435 The Oxford wind tunnel provides a different and complementary test environment

436 compared to other current Mars wind tunnels, such as the one at Aarhus University.

437 The most important difference is that the Oxford wind tunnel is designed to give

438 laminar rather than turbulent flow. The second important difference is that the

439 Oxford wind tunnel is open-circuit rather than closed-circuit. Re-circulating (closed-

440 circuit) wind tunnels offer a stable pressure and temperature environment; in contrast,

441 the temperature and pressure in the Oxford facility are a result of a dynamic

442 equilibrium. The disadvantage of this is that it is difficult to keep the temperature

443 stable with changes in flow speed and pressure. However, this dynamic balance

444 means that conditions can be changed very rapidly. Wind speed can be changed from

4450 to $30 \mathrm{~m} / \mathrm{s}$ (or vice versa) in $<1$ second, which allows testing of the response times

446 of meteorological instrumentation. The chamber pressure, too, can be changed

447 quickly: For example, it takes $\sim 10$ seconds to change the set-point from 6 mbar to 7

448 mbar. Although such rapid pressure changes are not representative of conditions on

449 Mars, it does mean that one can rapidly cycle through a set of test conditions. The 
450 temperature in the test section can also be rapidly varied because it too, as discussed

451 above, is a result of a dynamical equilibrium. If operating at a constant flow speed, it

452 takes several minutes to change the set temperature by, say, $10^{\circ} \mathrm{C}$, because of the

453 thermal mass of the heat exchanger. However, a rapid temperature change (e.g. $10^{\circ} \mathrm{C}$

454 in 10 seconds) can be obtained by altering the flow rate to change the thermal loads.

455 In conclusion, the dynamical balances of flow, pressure, and temperature in the open-

456 circuit Oxford Mars wind tunnel offer many opportunities for testing responses to

457 transients which are quite different, and complementary to, the stable environment of

458 a closed-circuit tunnel.

\section{$459 \quad 2.7$ Wind direction variation}

460 Although the test section of the wind tunnel is only $0.18 \mathrm{~m}$ in diameter (or $0.12 \mathrm{~m}$ if 461 using the smaller nozzle), the test chamber is $1.5 \mathrm{~m}$ in diameter, allowing plenty of 462 space for ancillary equipment such as rotation or translation stages. The most 463 frequent piece of equipment used is a motorised rotation stage used to change the 464 effective wind direction at the wind sensor. The test area is also large enough to 465 allow 2-D rotational stages to be used, e.g. to measure sensitivity to pitch and yaw 466 angles.

\section{Conclusions}

468 A wind tunnel optimised for testing of sensors in Martian pressures and wind speeds 469 has been designed and implemented. The wind tunnel provides laminar flow of air or 470 carbon dioxide at pressures of $5-10 \mathrm{mbar}$, temperatures of $200-300 \mathrm{~K}$, and speeds 471 of $0.5-30 \mathrm{~m} / \mathrm{s}$ (dependent on pressure and temperature selected). The nominal test 
472 section is $180 \mathrm{~mm}$ diameter, although a second nozzle of $120 \mathrm{~mm}$ diameter can also

473 be used to attain higher speeds (up to $\sim 60 \mathrm{~m} / \mathrm{s}$ ).

474 Detailed analytical and finite element modelling techniques have been used to 475 characterise the facility's flow characteristics. By measuring mass flow rate using a 476 set of orifice plates on the gas inlet, the Reynolds number of the flow in the test 477 section can be determined with an absolute accuracy of 2 to $3 \%$. Experimental 478 confirmation of flow profile predictions were obtained using a pitot probe for speeds 479 of above $\sim 5 \mathrm{~m} / \mathrm{s}$, and these show very good agreement with modelling predictions. 480 The absolute accuracy in flow speed of $\pm 3 \%$, and the stability of the flow to within $481 \pm 0.3 \%$, compare favourably with that achieved in other Mars wind sensor calibration 482 facilities. 


\section{Bibliography}

484 Barlow, J.B., 1999. Low-speed wind tunnel testing. Wiley, New York.

485 British Standard 1042, Measurement of fluid flow in closed conduits, Sections 1.1 (1981) and 486 1.4(1992). Also BS ISO TR 15377 (1998), BSI, London.

487 Chamberlain, T.E., Cole, H.L., Dutton, R.G., Greene, G.C., Tillman, J.E., 1976. Atmospheric 488 measurements on Mars: the Viking meteorology experiment. Bulletin of the American Meteorological 489 Society $57,1094-1104$.

490 Davey, R., Chamberlain, T., Harnett, L., 1973. Viking Meteorology Instrument Sensor Design 491 analysis report. TRW Systems Group document no. METC-021 (code ident. 11982), unpublished.

492 Dissly, R.W., Banfield, D.J., Lasnik, J., Waters, J.T., McEwan, I.J., Richardson, M.I., 2005.

493 Development of a Martian Sonic Anemometer. DPS meeting \#37, abstract \#18.05.

494 Evlanov, E.N., Zubkov, B.V., Nenarokov, D.F., Linkin, V.N., Zavjalov, M.A. \& Tyuryukanov, P.M., 495 2001. Gas-Discharge Anemometer for the Investigation of Flow Dynamics in Rarefied Gas Media. 496 Cosmic Research 39 (5), 484-490.

497 Fay, J.A. and Sonwalkar, N., 1991. Fluid Mechanics. MIT, Boston.

498 Greeley, R., Wilson, G., Coquilla, R., White, B., \& Haberle, B., 2000, Windblown dust on Mars:

499 laboratory simulations of flux as a function of surface roughness. Planetary and Space Science 48, (12-

$500 \quad 14)$, p $1349-1355$

501 Gunnlaugsson, H.P., Akinlade, O., Gross, M.A., Jensen, S.K., Lange, C.F., Leer, K., Lemmon, M.T.,

502 Lyckegard, F., Madsen, M.B., Merrison, J., Nørnberg, P., Olsen, M., Shinohara, C., Smith, P., 2006.

503 The Telltale Wind Experiment for the NASA Phoenix Mars Lander 2007. EGU general assembly

$504 \quad 2006$, abstract \#06064. 
505 Henry, R. M., Greene, G. C., 1974. Anemometers for Mars. In: Dowdell, R.B. (Ed.), Flow, its

506 measurement and control in science and industry. Instrument Society of America.

507 Merrison, J.P., Bertelsen, P, Frandsen, C., Gunnlaugsson, H.P., Knudsen, J.M., Lunt, S., Madsen, 508 M.B., Mossin, L.A., Nielsen, J., Nørnberg, P., Rasmussen, K.R., Uggerhøj, E., 2002. Simulation of the 509 Martian dust aerosol at low wind speeds. Journal of Geophysical Research, 107 (E12), 5133

510 Merrison, J.P., Gunnlaugsson, H.P., Kinch, K., Jacobsen, T.L., Jensen, A.E., Nornberg, P., Wahlgreen,

511 H., 2006. An integrated laser anemometer and dust accumulator for studying wind-induced dust 512 transport on Mars. Planetary and Space Science 54 (11), 1065-1072.

513 Parkinson, K.J., Day, W., 1979. The Use of Orifices to Control the Flow Rate of Gases. Journal of 514 Applied Ecology 16 (2), 623-632.

515 Seiff, A, Tillman, J.E., Murphy, J.R., Schofield, J.T., Crisp, D., Barnes, J.R., LaBaw, C., Mahoney, C., 516 Mihalov, J.D., Wilson, G.R., and Haberle, R.M., 1997. The atmosphere structure and meteorology 517 instrument on the Mars Pathfinder lander. Journal of Geophysical Research 102 (E2), 4045-4056.

518 Sullivan, R., Greeley, R., Kraft, M., Wilson, G., Golombek, M, Herkenhoff, K, Murphy, J., Smith, P., 519 2000. Results of the Imager for Mars Pathfinder windsock experiment. Journal of Geophysical 520 Research 105 (E10), 24547-24562.

521 White, B.R., Lacchia, B.M., Greeley, R., Leach, R.N., 1997. Aeolian behavior of dust in a simulated 522 Martian environment. Journal of Geophysical Research-Planets 102 (E11), 25629-25640.

523 Young, A. D., 1989. Boundary Layers $1^{\text {st }}$ edition. BSP Professional Books. 


\section{Figure Captions}

526 Figure 1 - A twenty-minute sequence of wind and temperature data measured by the Viking

527 Lander 2. The sampling interval for the data shown is 1.2 seconds. (Data provided by Jim 528 Murphy, NMSU).

$530 \quad$ Figure 2 - Three basic types of low density wind testing facility. In each diagram the grey 531 square indicates the position of the wind sensor (or test piece), the thick black lines represent 532 the walls of a vacuum chamber, and arrows represent airflow.

$534 \quad$ Figure 3 - Schematic diagram of wind tunnel

536 Figure 4 - Close-up of the test section of the wind tunnel, showing the nozzle and the 537 translation stage used to move the pitot tube through the flow field. The test chamber is 538 roughly $1.5 \mathrm{~m}$ diameter $\mathrm{x} 1.5 \mathrm{~m}$ in length.

540 Figure 5 - FEM calculations of flow profile in the $180 \mathrm{~mm}$ nozzle, calculated for carbon 541 dioxide at $p=6 \mathrm{mbar}$ and $T=250 \mathrm{~K}$.. At all velocities above $1 \mathrm{~m} / \mathrm{s}$ the velocity variation in 542 the central section (defined as $r<22.5 \mathrm{~mm}$ ) of the wind tunnel is $<1 \%$. At low velocities 543 the solution approaches the quadratic profile of Poiseuille flow (fully-developed laminar pipe 544 flow). Velocity at the centre of the test section is expressed as a proportion of $u_{\text {ave }}$ $545 \quad\left(u_{\text {ave }}=\int_{0}^{R} u(r) 2 \pi r d r\right)$. 
547 Figure 6 - Velocity profiles in the wind tunnel. Analytical and FEM results are presented, as

548 are experimental measurements obtained using the pitot probe. Profiles shown are average

549 speeds of 6.1 and $32.1 \mathrm{~m} / \mathrm{s}$, in air at $10 \mathrm{mbar}$ and $\mathrm{T}=299 \mathrm{~K}$; these flow profiles are

550 equivalent to what is obtained in $\mathrm{CO}_{2}$ at $250 \mathrm{~K}$ and 6 mbar at velocities of 3.8 and $19.7 \mathrm{~m} / \mathrm{s}$

551 respectively

552

553

554 Figure 7 -2D flow profile at test section, obtained using the pitot probe. The test gas was air 555 at $p_{\text {test }}=5.5 \mathrm{mbar}, T=293 \mathrm{~K}$.

557 Figure 8 - Time series of velocity measurements in the centre of the test section at two 558 different velocities, in air at 7 mbar, obtained using a thermal wind sensor (see text for 559 details). 
Viking Lander 2, sol 554
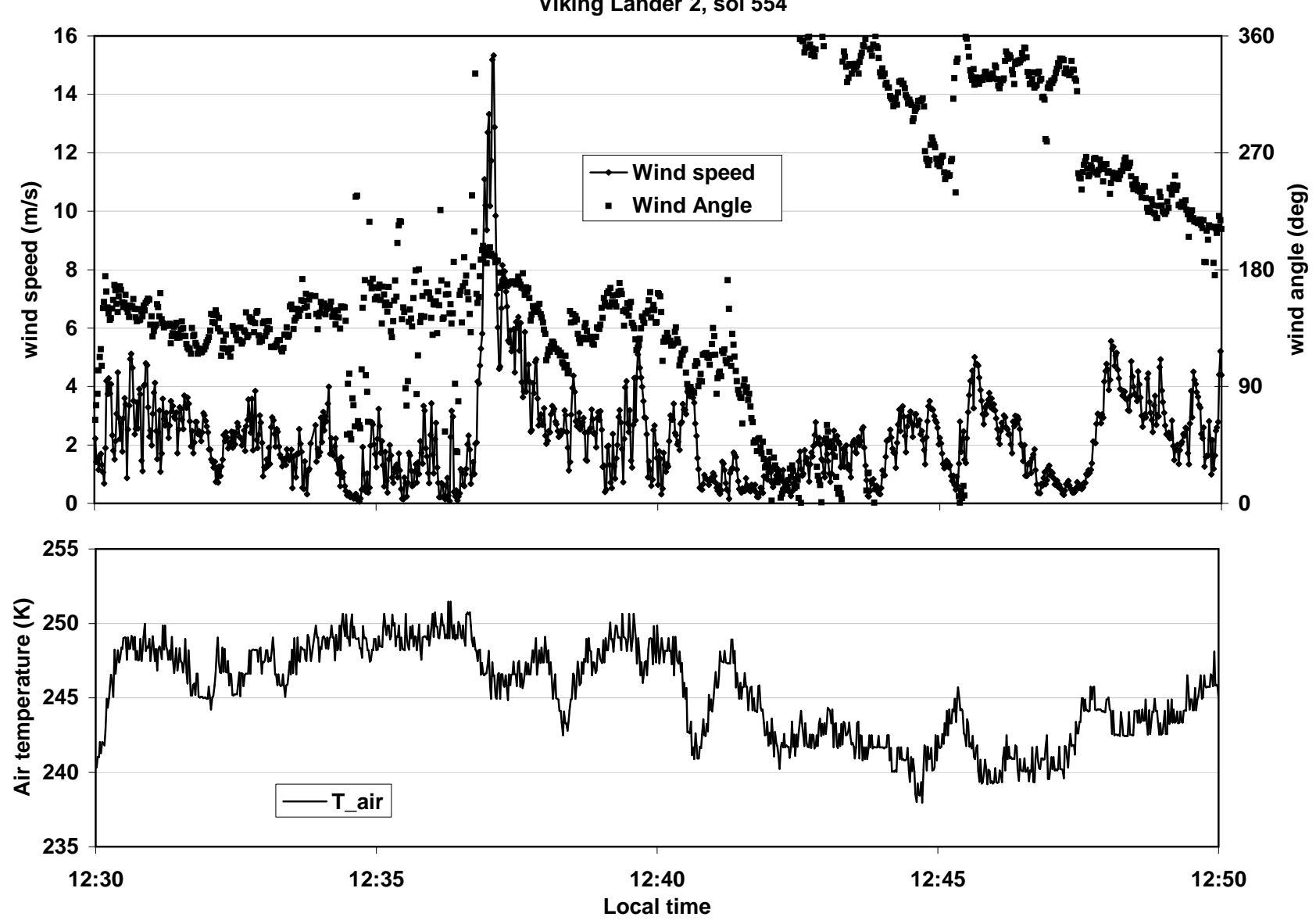
a) rotating arm

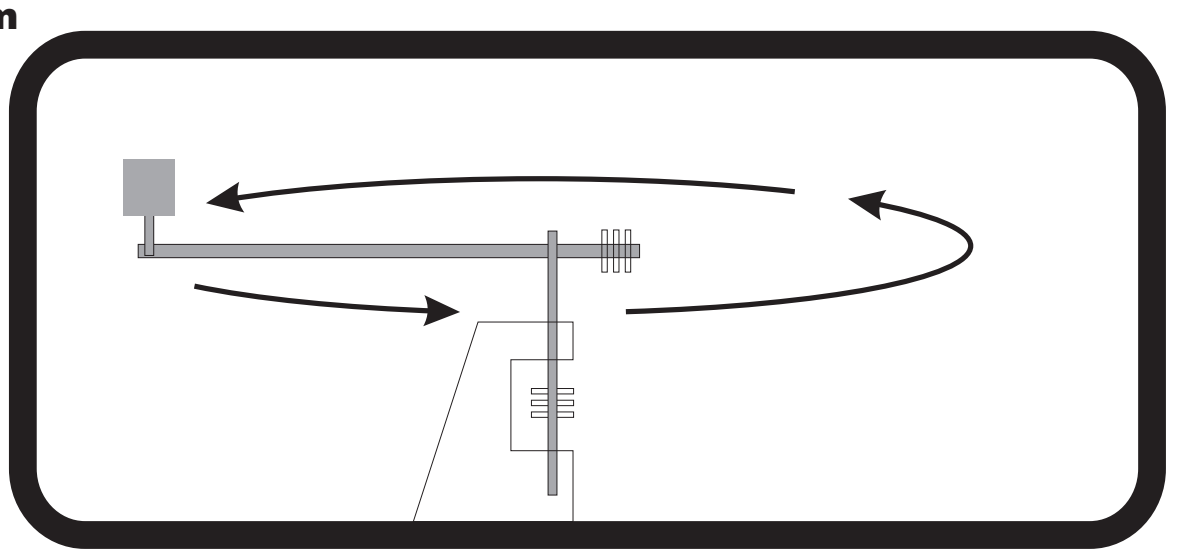

b) recirculating

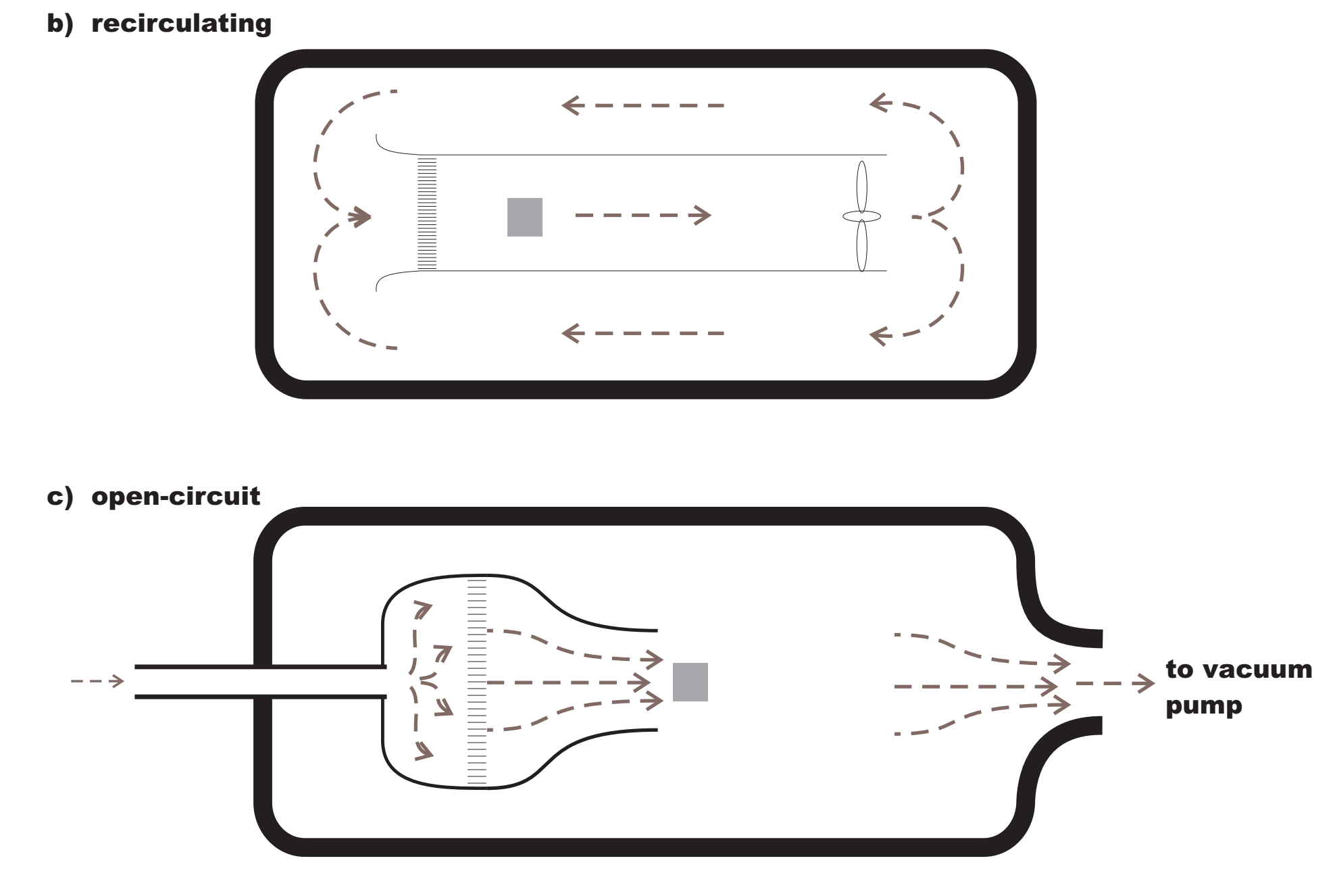

c) open-circuit

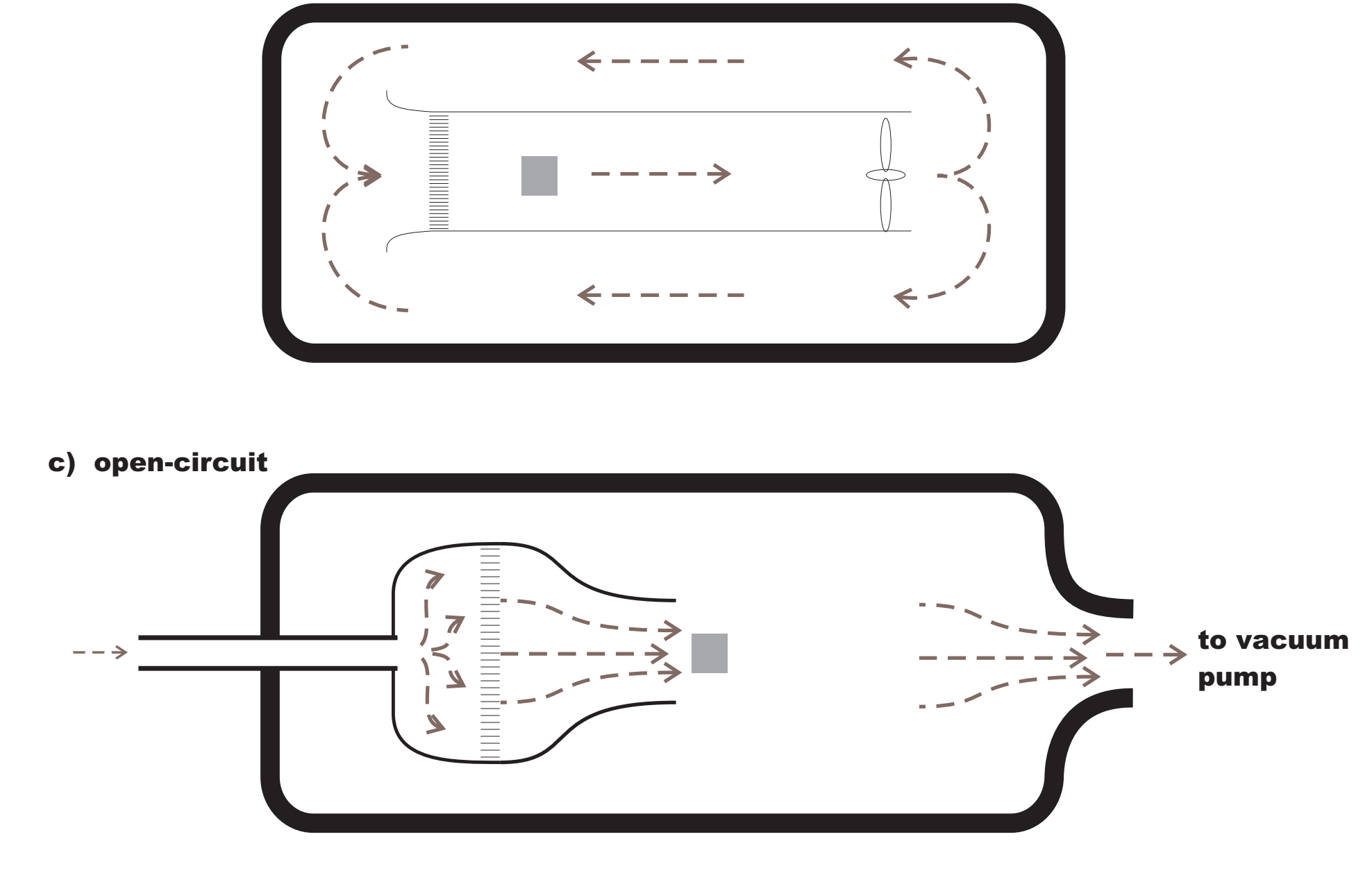

Figure 2

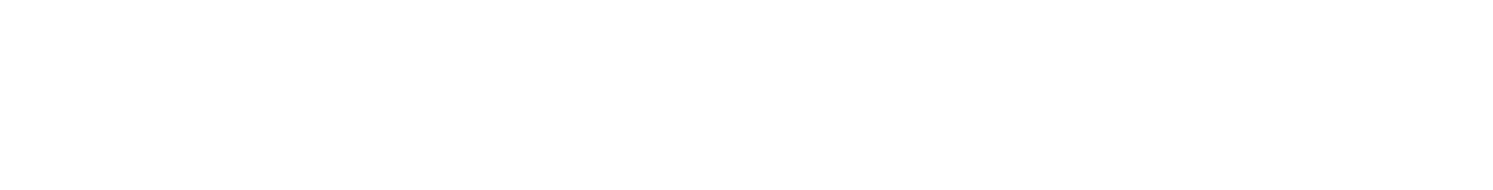


Figure 3

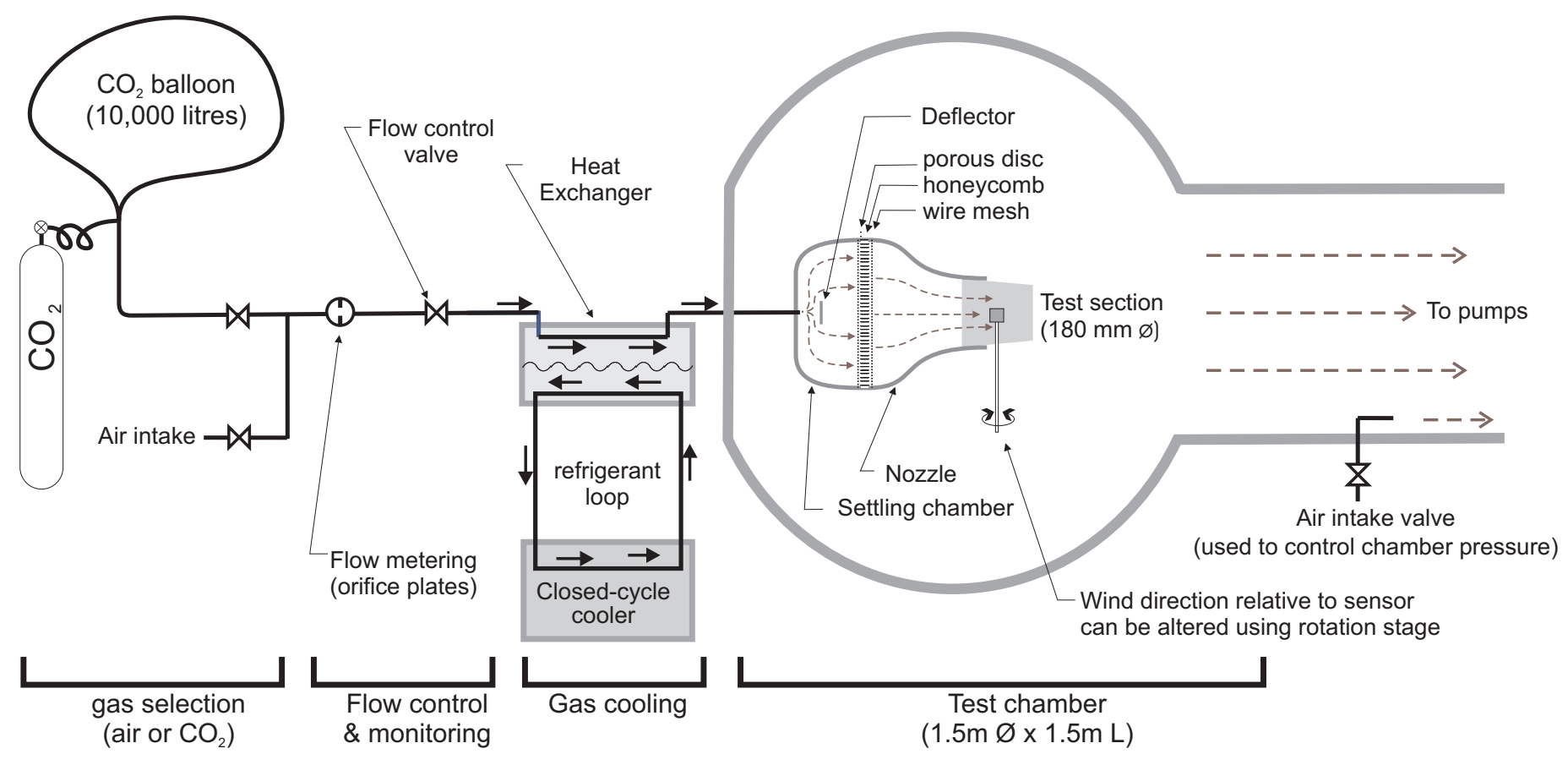




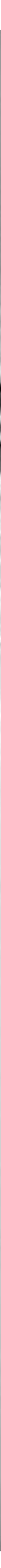




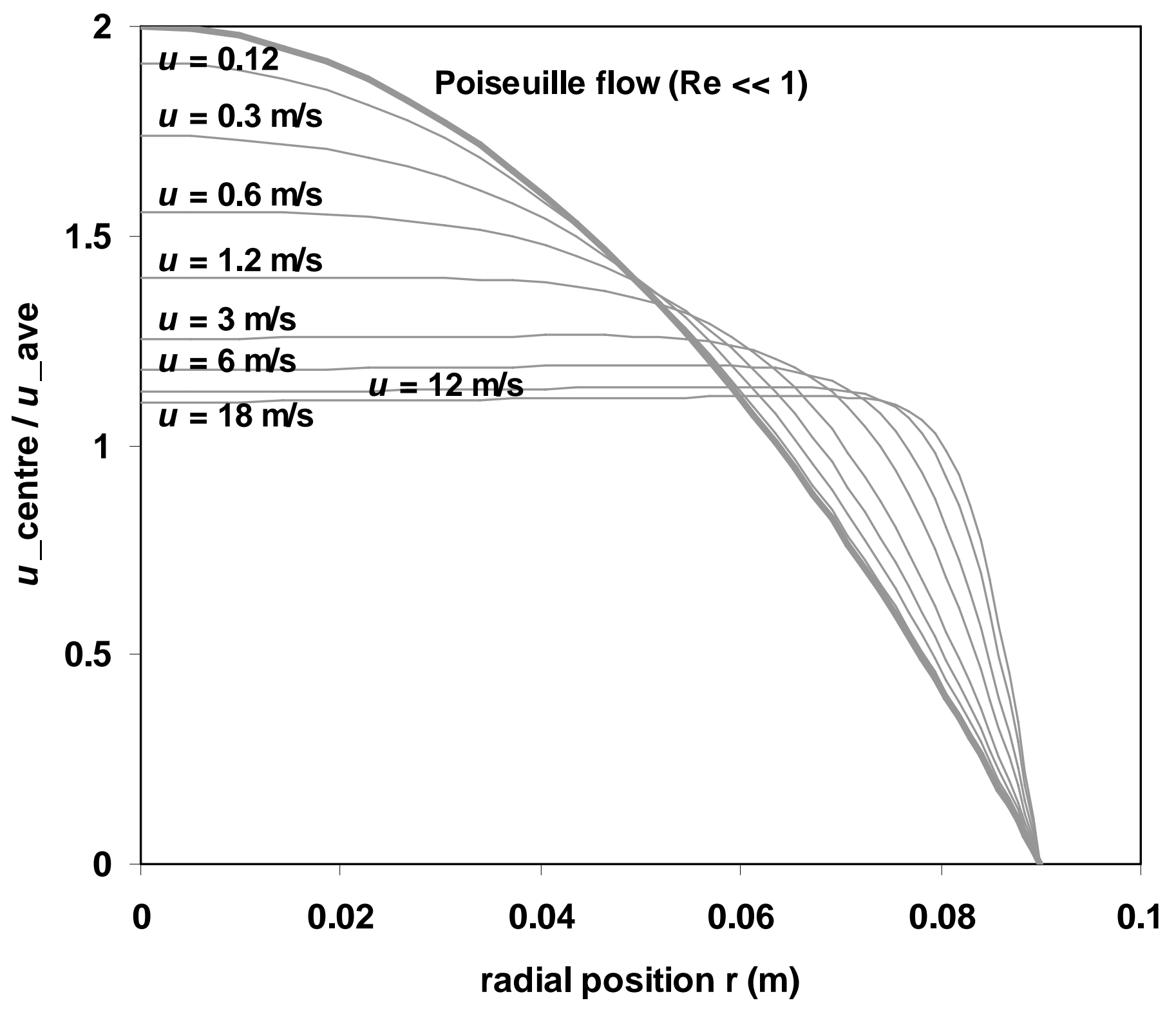



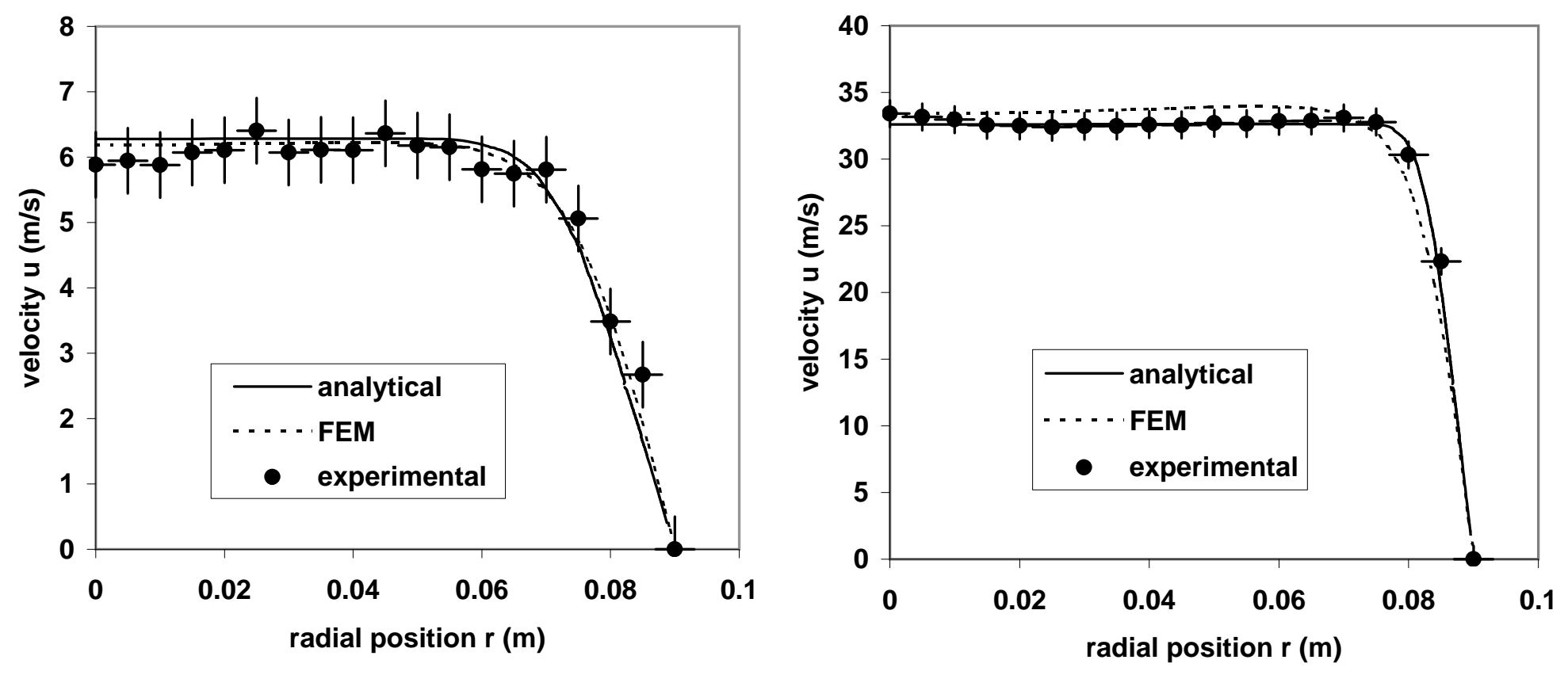


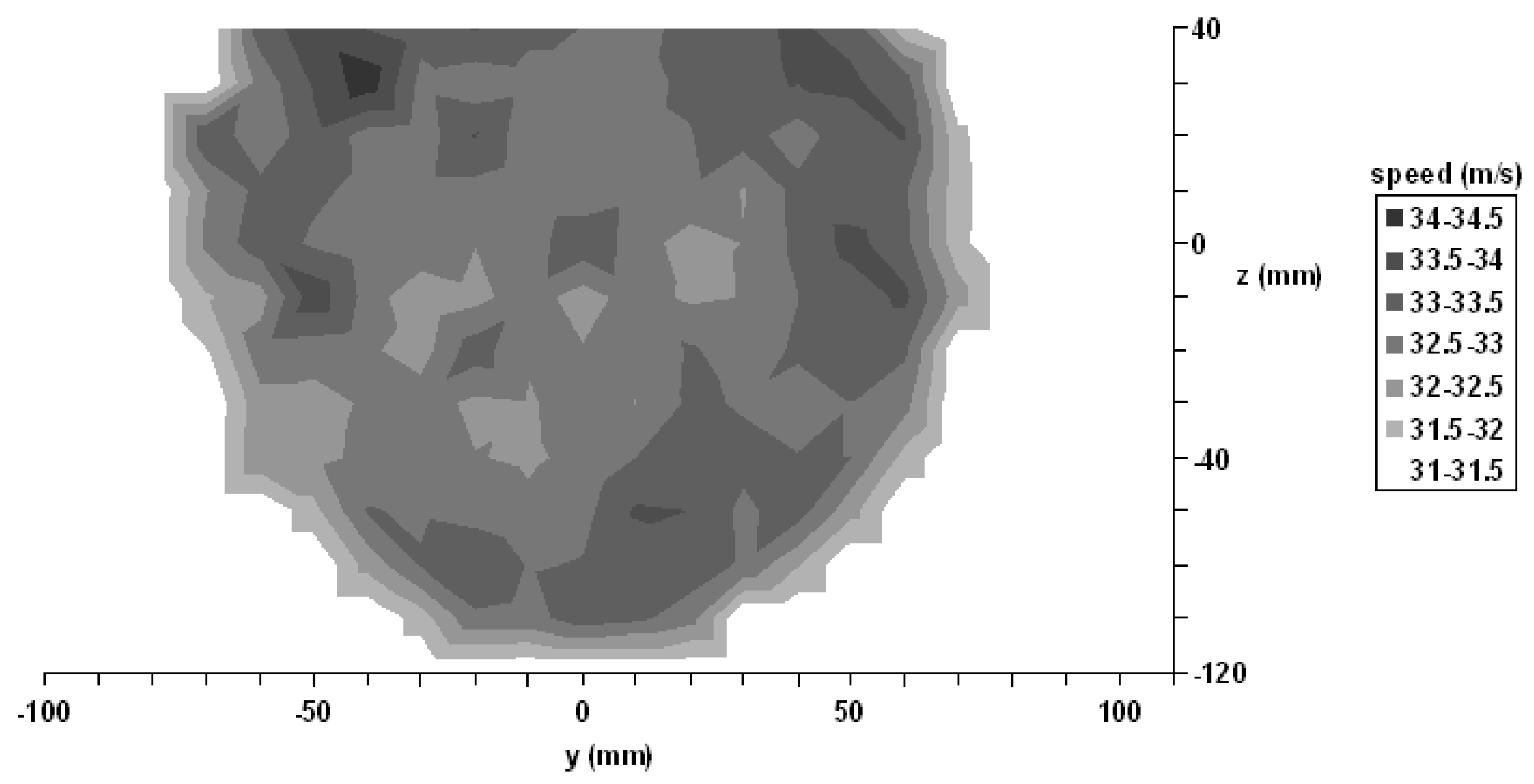


Figure 8
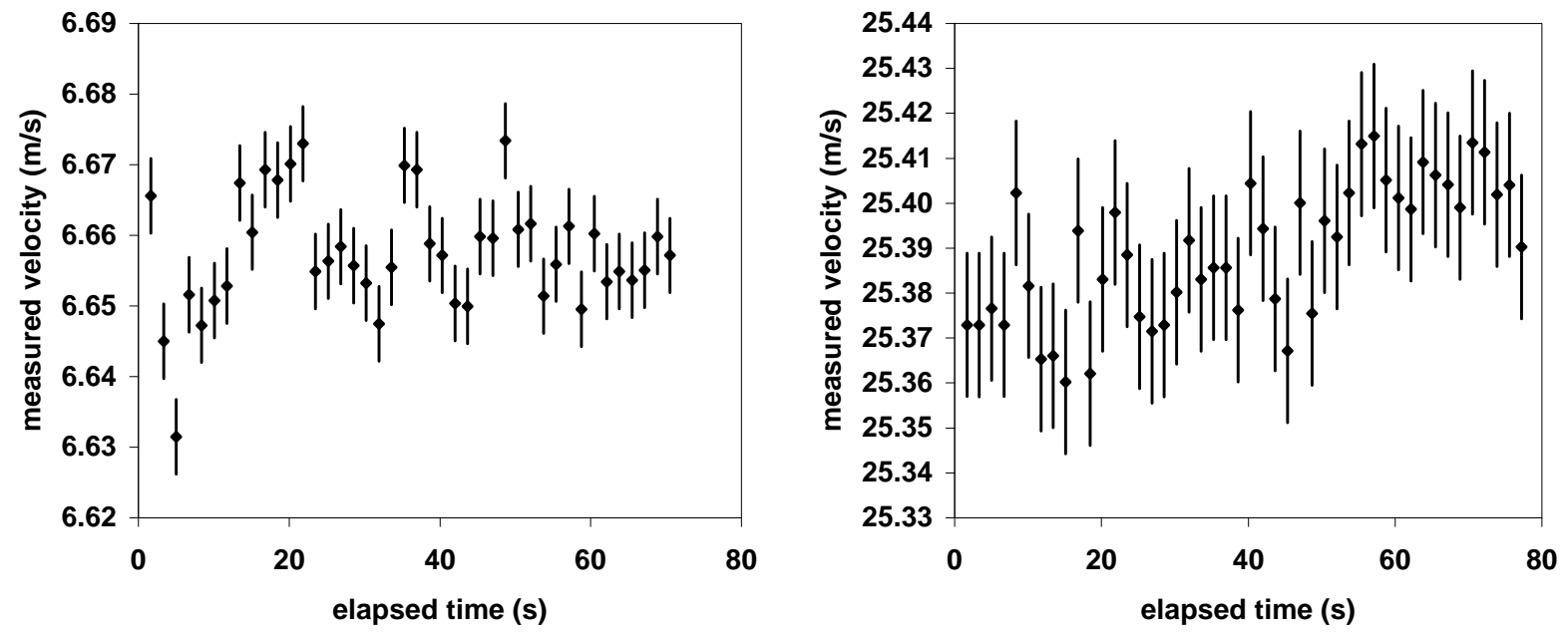